\title{
Dispersive Möbius Transform Finite-Element Time-Domain Method on Graphics Processing Units
}

\author{
David S. Abraham and Dennis D. Giannacopoulos \\ Department of Electrical and Computer Engineering, McGill University, Montréal, QC H3A 0E9, Canada
}

\begin{abstract}
A novel use of graphics processing units (GPUs) is presented in the execution of the dispersive finite-element time-domain (FETD) method, based upon the Möbius (bilinear) $z$-transform technique. By utilizing the immense computational power of modern GPUs via NVIDIA's compute unified device architecture (CUDA) language, a narrowing of the performance gap, which currently exists between dispersive FETD methods and their non-dispersive counterparts, can be achieved, thus facilitating the study of a wider range of physical phenomena. An analysis of the $z$-transform dispersive FETD algorithm is presented in order to both identify dispersive overhead bottlenecks and determine its suitability to parallelization. Numerical studies are then undertaken to measure the performance increase as a function of simulation parameters, such as number of variables, the amount of dispersive material present, and floating point precision.
\end{abstract}

Index Terms-Dispersive media, finite-element time-domain (FETD) method, graphics processing unit (GPU), parallel processing.

\section{INTRODUCTION}

O VER THE past several years, a growing amount of research has been devoted to the study of frequencydependent media and their numerical treatment in electromagnetics simulations. While all real-world materials exhibit some kind of dispersion, often the effect is small enough as to be neglected over a narrow frequency range of interest, allowing for an excellent treatment via existing and wellestablished numerical techniques, such as the finite-element time-domain (FETD) method [1]. Nonetheless, such simplifications are not always possible, particularly when dealing with wideband electromagnetics simulations or for problems in which a higher degree of accuracy is required [2]. In such circumstances, a proper dispersive time-domain treatment is required in order to capture the full response of the system. Such methods have far reaching applications, from the study of dispersive human tissue samples in medical imaging [3], to dispersive environmental elements in radar applications [4].

Given the clear need for dispersive time-domain solvers, several extensions to the FETD method have been proposed, being broadly characterized into three distinct groups: 1) recursive convolution [2]; 2) auxiliary differential equation [5]; and 3) Möbius z-transform [6]. While these first two methods have achieved notable success, the treatment of arbitrarily high order phenomena can rapidly become intractable. In contrast, the $z$-transform method converts material parameters to the $z$-domain, allowing for the derivation of update equations that remain relatively simple and efficient at arbitrarily high dispersive orders.

Despite improvements in efficiency, dispersive methods have naturally remained, in general, slower than their non-dispersive counterparts. In many cases, the additional

Manuscript received July 6, 2015; revised September 4, 2015; accepted September 30, 2015. Date of publication October 8, 2015; date of current version February 17, 2016. Corresponding author: D. D. Giannacopoulos (e-mail: dennis.giannacopoulos@mcgill.ca).

Color versions of one or more of the figures in this paper are available online at http://ieeexplore.ieee.org.

Digital Object Identifier 10.1109/TMAG.2015.2488641 inescapable complexity imposed by dispersive media can constitute a large and debilitating overhead, hampering analysis and design efforts.

The goal of this paper, therefore, is to attempt to narrow the performance gap which currently exists between dispersive and non-dispersive FETD $z$-transform methods, through the use of graphics processing units (GPUs) and their massively parallel architectures. While many aspects of the standard FETD algorithm [7], as well as dispersive finite-difference time-domain methods [8], have already seen GPU implementations, little work has been done in addressing the additional complexity inherited by the FETD methods due to dispersion. The innovation of this paper, therefore, is the isolation and acceleration of additional overhead imposed specifically, by dispersive elements within the relatively simpler and more accurate $z$-transform formulation. As such, the results obtained herein can be easily coupled to the aforementioned existing GPU implementations of the base scheme, for even greater computational efficiency.

In doing so, it is hoped that dispersive methods may be rendered far more efficient, allowing for an ever more accurate characterization of physical phenomena, without debilitating overhead.

\section{Möbius (BIlinear) Transform Method}

The standard FETD formulation for the second-order vector wave equation (such as in [1]), forms the basis of the bilinear transform method. The inclusion of dispersion necessitates the introduction of convolutions between the fields and the material parameters within the FETD equations, resulting in the following system of ordinary differential equations in time [6]:

$$
[T] \epsilon(t) * \frac{d^{2}\{u\}}{d t^{2}}+[S] \frac{1}{\mu(t)} *\{u\}+\{f\}=\{0\}
$$

where $[T]$ and $[S]$ represent the mass and stiffness matrices, $\{f\}$ is the source term related to the electric current density, and $\epsilon(t)$ and $\mu(t)$ are the permittivity and the permeability. The Newmark- $\beta$ scheme can then be applied in order to 
discretize the time derivatives; however, this would naturally require temporal discretizations of terms of the form $\epsilon(t) *[T]\{u\}(t)$ and $\mu^{-1}(t) *[S]\{u\}(t)$, denoted by $\left\{\mathcal{L}_{\epsilon}\right\}(t)$ and $\left\{\mathcal{L}_{\mu^{-1}}\right\}(t)$, respectively. To obtain update equations for these convolutions, the $z$-transform method derived in [6] is now briefly summarized.

The vast majority of the dispersive materials commonly encountered in electromagnetics have material parameters that can be expressed as rational functions in the Laplace domain [1]. A bilinear transformation (a special case of the Möbius transform) of the following form may then be applied:

$$
s \mapsto \frac{2}{\Delta t} \frac{1-z^{-1}}{1+z^{-1}}
$$

where $\Delta t$ is the discrete time step used in the Newmark- $\beta$ scheme. This maps the material parameters to the $z$-domain, allowing for the exploitation of two crucial properties of the $z$-transform: 1) convolution/multiplication and 2) time shifting. Applying these properties to $\left\{\mathcal{L}_{\epsilon}\right\}$ and $\left\{\mathcal{L}_{\mu^{-1}}\right\}$ in the $z$-domain allows for the derivation of update equations to march the convolutions forward in time, in concert with the solution.

Continuing to follow [6], the transposed direct form II technique is adopted from signal processing, allowing for the previous field and the convolution values to be accumulated in auxiliary variables rather than explicitly stored, yielding the following update equations per element for $\left\{\mathcal{L}_{\epsilon}\right\}$, with those for $\left\{\mathcal{L}_{\mu^{-1}}\right\}$ being obtained similarly:

$$
\begin{aligned}
\left\{\mathcal{L}_{\epsilon}\right\}^{n+1} & =c_{0}[T]\{u\}^{n+1}+\left\{\mathcal{W}_{1}\right\}^{n} \\
\left\{\mathcal{W}_{\alpha}\right\}^{n+1} & =c_{\alpha}[T]\{u\}^{n+1}-d_{\alpha}\left\{\mathcal{L}_{\epsilon}\right\}^{n+1}+\left\{\mathcal{W}_{\alpha+1}\right\}^{n}, \quad \alpha<p
\end{aligned}
$$

$$
\left\{\mathcal{W}_{\alpha}\right\}^{n+1}=c_{\alpha}[T]\{u\}^{n+1}-d_{\alpha}\left\{\mathcal{L}_{\epsilon}\right\}^{n+1}, \quad \alpha=p
$$

in which $\left\{\mathcal{W}_{\alpha}\right\}$ (and $\left\{\mathcal{G}_{\alpha}\right\}$ in the magnetic case) are the auxiliary variables in question, $c_{\alpha}$ and $d_{\alpha}$ are parameters associated with the medium's dispersive model, and $p$ is the order of dispersion. The present value of the convolution is, therefore, dependent on past field and convolution values, with the amount of history required depending on $p$.

Combining these auxiliary variables with the Newmark- $\beta$ discretization of (1) with $\beta=1 / 4$ then allows the FETD update equations to take on the following form:

$$
\begin{aligned}
& \left(\frac{1}{\Delta t^{2}}[T]+\frac{1}{4}[S]\right)\{u\}^{n+1}=\left(\frac{2}{\Delta t^{2}}[T]-\frac{1}{2}[S]\right)\{u\}^{n} \\
& -\left(\frac{1}{\Delta t^{2}}[T]+\frac{1}{4}[S]\right)\{u\}^{n-1}-\frac{1}{\Delta t^{2}}\left(\{\mathcal{W}\}^{n}-2\{\mathcal{W}\}^{n-1}+\{\mathcal{W}\}^{n-2}\right) \\
& -\frac{1}{4}\left(\{\mathcal{G}\}^{n}+2\{\mathcal{G}\}^{n-1}+\{\mathcal{G}\}^{n-2}\right) \\
& -\left(\frac{1}{4}\{f\}^{n+1}+\frac{1}{2}\{f\}^{n}+\frac{1}{4}\{f\}^{n-1}\right)
\end{aligned}
$$

in which $\{\mathcal{W}\}$ and $\{\mathcal{G}\}$ are global vectors assembled from the elemental $\left\{\mathcal{W}_{1}\right\}$ and $\left\{\mathcal{G}_{1}\right\}$ vectors. For a full treatment and derivation of the above method see [6].

Upon comparing (6) with the standard FETD update equation, the only significant deviation is found in the inclusion of the auxiliary variables. As such, the incorporation of dispersion within the $z$-transform dispersive FETD method is tantamount to the evaluation of (3)-(5) within each iteration. It is these update equations which form the bulk of the dispersive overhead and will now become the focus of parallelization.

\section{Parallelization Strategy}

The compute unified device architecture (CUDA) language introduced by NVIDIA in 2006 revolutionized GPU utilization in scientific computing, allowing programmers unprecedented access to NVIDIA hardware [9]. The basis of CUDA, in essence, is the grouping of threads into units or warps which execute code simultaneously in lock step with each other, with the ability to work off different data sets and to branch independently, if need be [10]. This single-instruction multiple thread (SIMT) system, thereby allows for high computational throughput and flexibility, particularly when dealing with problems exhibiting a high degree of repetition and independence [9].

In investigating (3)-(5), it is important to note that all the required update procedures are composed of three elementary linear algebra operations: 1) matrix-vector product; 2) vectorscalar product; and 3) vector addition. Expressing the product between $[T]$ and $\{u\}^{n+1}$ by definition as a summation, as in

$$
\left\{[T]\{u\}^{n+1}\right\}_{i}=\sum_{j=1}^{n}[T]_{i, j}\{u\}_{j}^{n+1}
$$

reveals how each $i$ 's operations are identical and independent, simply executed on different data sets. Furthermore, expanding the scaling and addition operations in like manner reveals a similar independent structure, as in

$$
\left\{\mathcal{W}_{\alpha}\right\}_{i}^{n+1}=c_{\alpha}\{[T]\{u\}\}_{i}^{n+1}-d_{\alpha}\left\{\mathcal{L}_{\epsilon}\right\}_{i}^{n+1}+\left\{\mathcal{W}_{\alpha+1}\right\}_{i}^{n} .
$$

Hence, it is concluded that the above operations are excellent candidates for implementation in the CUDA language, due to their adherence to the SIMT and independence principles.

Since the scaling and addition operations of (8) are dependent on the product of (7), however, the two operations cannot occur concurrently. This suggests that each thread or worker on the GPU be responsible for the computation of the $i$ th entry of the product vector and the subsequent scaling and additions for each auxiliary variable. In this manner, each entry of the auxiliary variables in (3)-(5) may be updated concurrently, thus improving efficiency. This proposed algorithm is shown in Fig. 1 for an electrically dispersive medium in which $\{\mathcal{G}\}$ has been set to zero.

The use of the GPU does, however, come with its own potential overhead. The GPU and host device do not share the same physical memory, and so it is necessary to transfer data between the two. Since the present investigation is concerned only with the parallelization of the dispersive overhead, intimate cooperation with the CPU for steps such as matrix solving are still required, as the base FETD method is not here parallelized [although doing so can yield additional benefits (see Fig. 4)]. From (3)-(5), it is clear that knowledge of $\{u\}^{n+1}$ is needed by the GPU to update all auxiliary variables, while the combination $\{\mathcal{K}\}=\left(1 / \Delta t^{2}\right)\left(\{\mathcal{W}\}^{n}-2\{\mathcal{W}\}^{n-1}+\right.$ $\left.\{\mathcal{W}\}^{n-2}\right)-(1 / 4)\left(\{\mathcal{G}\}^{n}+2\{\mathcal{G}\}^{n-1}+\{\mathcal{G}\}^{n-2}\right)$ is needed by the 


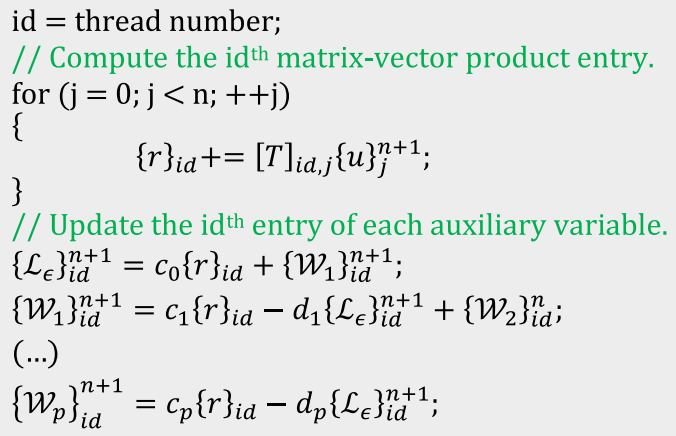

Fig. 1. Parallelized pseudocode to update the auxiliary variables for an electrically dispersive medium.

CPU for the next iteration. The interaction between the CPU and the GPU is shown in Fig. 2, wherein it becomes clear that any performance increase granted by the GPU will cause the dispersive method's execution to asymptotically approach that of the non-dispersive method.

To obtain the best possible results on the part of the GPU, details of its architecture and memory also need to be considered during programming. For example, it is of the utmost importance that all GPU memory accesses be coalesced for optimal performance [10], as CUDA allows for whole memory blocks to be fetched in a single transfer, rather than individually [9]. As a result, the matrix data of (3)-(5) should be stored in a column major format, and all vectors held contiguously in memory [11].

\section{RESUlts}

A test problem was selected to which the above analysis could then be applied, and performance measured. Owing to its simplicity, and the fact that performance is tied mainly to the amount of dispersive elements and not their physical configuration, a 2-D parallel plate waveguide was selected measuring $20 \mathrm{~cm} \times 4 \mathrm{~cm}$, in which the upper and lower plates constituted perfect electric conductors, with each end of the waveguide fitted with a first-order absorbing boundary condition. A $4 \mathrm{~cm}$ tall doubly dispersive fourth-order dielectric slab was present, such that the total amount of dispersive material varied lengthwise between $25 \%$ and $90 \%$ of the total volume, with the remainder being free space. All computations were performed on the McGill high performance computing cluster, also known as Guillimin [12]. Control computations were made with a traditional serial CPU version of the algorithm, executed on one of Guillimin's 216 SW2 nodes equipped with dual Intel Sandy Bridge EP E5-2670 processors, clocked at $2.6 \mathrm{GHz}$, with $4 \mathrm{~GB}$ of RAM per core. This was compared with a parallelized version as in Fig. 2, whose code was run on one of Guillimin's AW accelerator nodes, containing the same Intel processors, as well as dedicated dual NVIDIA K20 graphics cards, clocked at $706 \mathrm{MHz}$ with $5 \mathrm{~GB}$ of dedicated video memory, and 2496 CUDA cores.

In order to first put into perspective the significance of the dispersive overhead, Table I is presented in which the percent of total execution time spent updating the auxiliary variables
(...)
$\{u\}^{n+1}=[A]^{-1}\{b\}$
// Compute $[A]$ and $\{\mathrm{b}\}$
cudaMemcpy(
$<<<$ Kernel $>>>$
// Compute $\{u\}^{n+1}$.
cudaMemcpy()
// Transfer $\{u\}$ from CPU to GPU.
(..)
// Update auxiliary variables.
// Transfer $\{\mathcal{K}\}$ back to CPU.
// Repeat the process.

Fig. 2. Main program loop pseudocode for parallelized dispersive FETD method.

TABLE I

Dispersive Overhead as a Percent of Total Computation Time

\begin{tabular}{ccccc}
\hline \hline Number of & \multicolumn{4}{c}{ Proportion of Dispersive Material } \\
Variables & $25 \%$ & $50 \%$ & $75 \%$ & $90 \%$ \\
\hline 23,840 & $9.85 \%$ & $17.80 \%$ & $24.29 \%$ & $27.88 \%$ \\
95,680 & $8.28 \%$ & $20.32 \%$ & $27.51 \%$ & $31.11 \%$ \\
383,360 & $10.28 \%$ & $19.04 \%$ & $25.89 \%$ & $29.03 \%$ \\
$1,534,720$ & $9.54 \%$ & $17.66 \%$ & $23.51 \%$ & $27.55 \%$ \\
\hline \hline
\end{tabular}

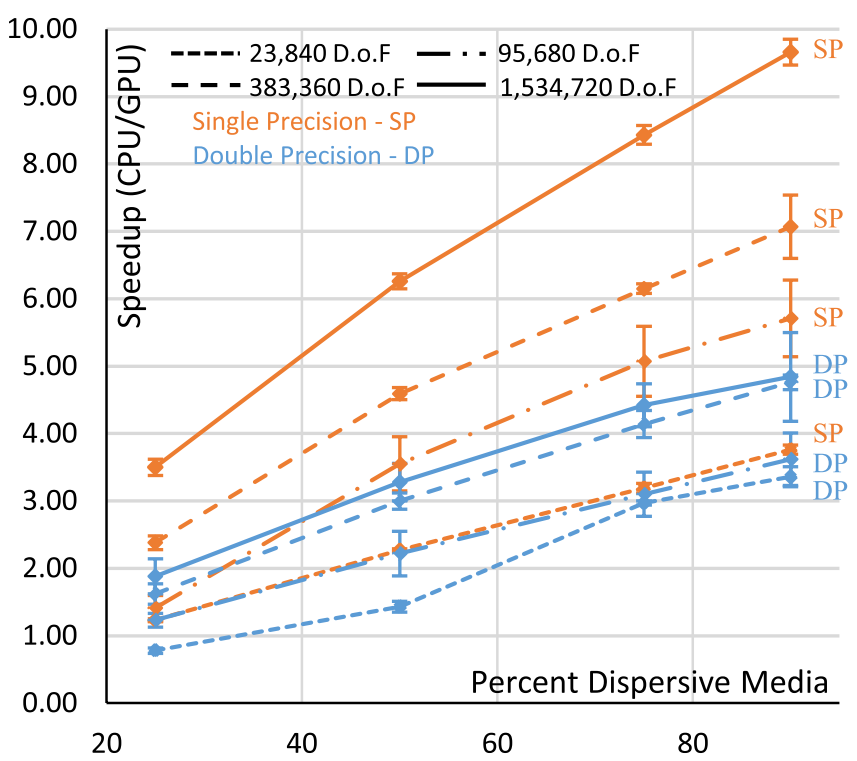

Fig. 3. GPU speedup as a function of problem size, floating point precision, and the amount of dispersive material, for a doubly dispersive, fourth order problem.

(for a traditional serial CPU implementation) is reported as a function of the amount of dispersive material and problem size, for 6000 time steps.

From these data, it is clear that the greatest benefit to be gained via parallelization is in high order, doubly dispersive problems for which a significant amount of dispersive material is present. Low order and/or singly dispersive materials may also experience benefit in parallelization, though the magnitude of any overall savings may be substantially less, depending on the problem. Whether the present algorithm is worthwhile to implement depends partly, therefore, on the specific problem under consideration, as those containing little dispersive material with relatively simple frequency dependence do not differ greatly in performance from non-dispersive media. 


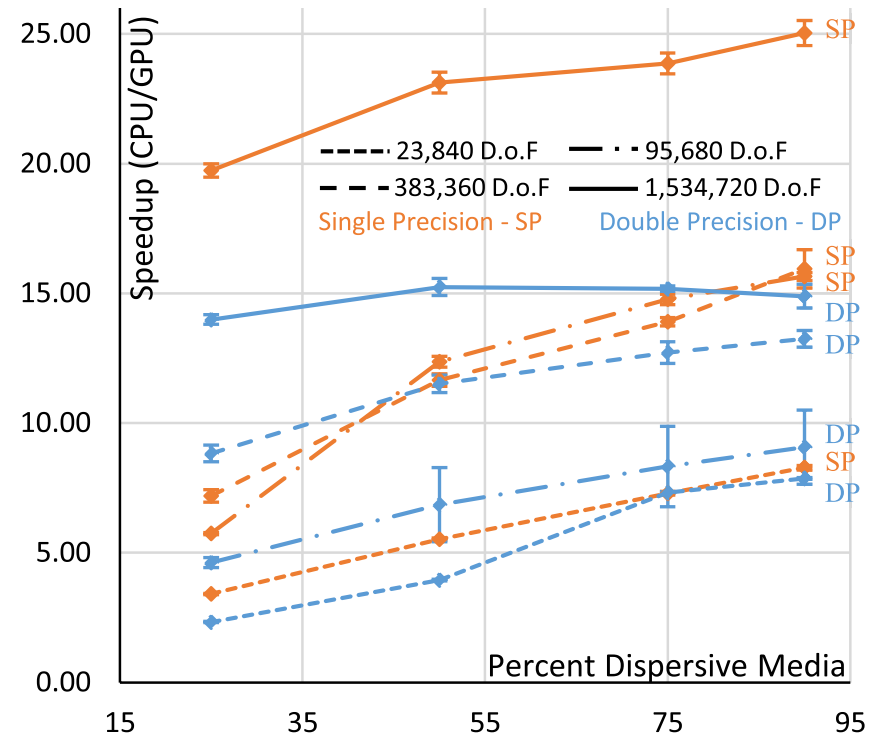

Fig. 4. Recreation of Fig. 3 in which the performance metric has been altered to exclude memory transfers between the CPU and the GPU.

The metric of choice for evaluating the effectiveness of the GPU was that of (9), and includes the overhead associated with the GPU memory transfers. As a result, Fig. 3 shows the GPU speedup as a function of problem size, floating point precision, and the amount of dispersive material present. Each simulation was run ten times and the results averaged, with error bars representing plus or minus one standard deviation from the mean.

The GPU performs very well, with a peak performance 9.66 times faster than the serial CPU version. Furthermore, performance can be seen to increase with the number of degrees of freedom, as well as the amount of dispersive material present. This is to be expected, as large problems with high quantities of dispersive elements have greater computational workload. This allows for increased utilization of GPU resources, better latency hiding, and greater opportunity for instruction level parallelism, resulting in increased performance [10]. Finally, it is also noted that the single precision analyses are far faster than their double precision counterparts, an expected result given the greater complexity of the latter.

While Fig. 3 shows good performance increase, a

$$
S_{p}=\frac{\text { Time Updating Auxiliary Variables on CPU }}{\text { Total Time on GPU }}
$$

detailed breakdown of GPU execution reveals that in most cases, more than $60 \%$ of time spent on the GPU was due to memory transfer overheads. This suggests that any implementation of the above algorithm should strive to reduce or altogether eliminate memory transfers between the host and the GPU during each iteration. This could be accomplished in the future by integrating the present method into the existing parallelizations of the base FETD scheme, particularly methods, such as [13], in which additional parallelism is exposed at the element level. In this way, matrix solving and variable updating may all take place on the GPU, ensuring that once data are placed in GPU global memory, it stays there. Indeed, if the performance metric (9) is altered to exclude memory transfer times, performance can be seen to be almost tripled, as shown in Fig. 4.

\section{CONClusion}

In conclusion, the overhead inherited by the FETD methods due to the presence of dispersion within the $z$-transform formulation was found to be an excellent candidate for parallelization through GPUs. The GPU performed extremely well, executing the auxiliary variable update operations at a peak rate of 9.66 times faster than a traditional CPU. When the burden of GPU memory transfers is omitted, the peak performance was found to jump to 25.04 times faster, strongly motivating future implementations to integrate this paper into the existing parallelizations of the base FETD algorithm.

\section{ACKNOWLEDGMENT}

Computations were made on the supercomputer Guillimin from McGill University, managed by Calcul Québec and Compute Canada. This work was supported in part by the Centre for Innovation, in part by NanoQuébec, in part by Réseau de médecine génétique appliquée, and in part by Fonds de recherche du Québec-Nature et technologies, for their funding of the supercomputer.

\section{REFERENCES}

[1] J.-M. Jin, The Finite Element Method in Electromagnetics, 2nd ed. New York, NY, USA: Wiley, 2002.

[2] D. Jiao and J.-M. Jin, "Time-domain finite-element modeling of dispersive media," IEEE Microw. Wireless Compon. Lett., vol. 11, no. 5, pp. 220-222, May 2001.

[3] C. Rappaport, "A dispersive microwave model for human breast tissue suitable for FDTD computation," IEEE Antennas Wireless Propag. Lett., vol. 6, no. 1, pp. 179-181, Apr. 2007.

[4] F. L. Teixeira, W. C. Chew, M. Straka, M. L. Oristaglio, and T. Wang, "Finite-difference time-domain simulation of ground penetrating radar on dispersive, inhomogeneous, and conductive soils," IEEE Trans. Geosci. Remote Sens., vol. 36, no. 6, pp. 1928-1937, Nov. 1998.

[5] F. L. Teixeira, "Time-domain finite-difference and finite-element methods for Maxwell equations in complex media," IEEE Trans. Antennas Propag., vol. 56, no. 8, pp. 2150-2166, Aug. 2008.

[6] A. Akbarzadeh-Sharbaf and D. D. Giannacopoulos, "A stable and efficient direct time integration of the vector wave equation in the finite-element time-domain method for dispersive media," IEEE Trans. Antennas Propag., vol. 63, no. 1, pp. 314-321, Jan. 2015.

[7] H.-T. Meng, B.-L. Nie, S. Wong, C. Macon, and J.-M. Jin, "GPU accelerated finite-element computation for electromagnetic analysis," IEEE Antennas Propag. Mag., vol. 56, no. 2, pp. 39-62, Apr. 2014

[8] M. R. Zunoubi, J. Payne, and W. P. Roach, "CUDA implementation of $\mathrm{TE}^{z}$-FDTD solution of Maxwell's equations in dispersive media," IEEE Antennas Wireless Propag. Lett., vol. 9, no. 1, pp. 756-759, Jul. 2010.

[9] "CUDA C programming guide," NVIDIA Corporation, Santa Clara, CA, USA, Tech. Rep. PG-02829-001_v6.5, Aug. 2014.

[10] N. Wilt, The CUDA Handbook. Upper Saddle River, NJ, USA: Addison-Wesley, 2013.

[11] N. Bell and M. Garland, "Efficient sparse matrix-vector multiplication on CUDA," NVIDIA Corporation, Santa Clara, CA, USA, Tech. Rep. NVR-2008-004, Dec. 2008.

[12] McGill HPC. (2013). Guillimin Hardware Information. [Online]. Available: http://www.hpc.mcgill.ca/index.php/starthere/81-doc-pages/ 215-guillimin-hardware, accessed May 11, 2015.

[13] D. M. Fernández, M. M. Dehnavi, W. J. Gross, and D. Giannacopoulos, "Alternate parallel processing approach for FEM," IEEE Trans. Magn., vol. 48, no. 2, pp. 399-402, Feb. 2012. 